\title{
Substance Use Completion Status
}

National Cancer Institute

\section{Source}

National Cancer Institute. Substance Use Completion Status. NCI Thesaurus. Code C162337.

A term used to describe the state or condition of the completeness of the substance use data. 